\title{
GW23-e1166 DRUG INTERACTION STUDIES OF ATORVASTATIN AND OTHER DRUGS BASED ON BIOINFORMATICS
}

doi:10.1136/heartjnl-2012-302920a.46

${ }^{1}$ Yan Gao, ${ }^{2}$ Li Fan, ${ }^{3}$ Xue-Chun Lu, ${ }^{2}$ Jian Cao, ${ }^{1}$ Xia Zhao, ${ }^{1}$ Jing Pei, ${ }^{1}$ Xiao-yan Meng, ${ }^{4}$ Li Fan. ${ }^{1}$ Third Department of Cadre Ward, General Hospital of Jinan Military Region; ${ }^{2}$ First Geriatric Cardiology Division, PLA General Hospital; ${ }^{3}$ Department of Geriatric Hematology, PLA General Hospital; ${ }^{4}$ First Geriatric Cardiology Division, Chinese PLA General Hospital

Objectives To explore the drug interactions between atorvastatin and other drugs based on bioinformatics.

Methods The human umbilical vein endothelial cell line EA. hy 926 cultured in vitro was incubated with atorvastatin $\left(10^{-5} \mathrm{~mol} / \mathrm{l}\right)$ for $24 \mathrm{~h}$. Total RNA from these cells was extracted and performed for gene expression profiling. The SAM microarray analysis software was used to analyse the differenced genes between the control and experiment groups. Gene set enrichment analysis (GSEA) was used for pathway analysis, and gene ontology (GO) analysis. The Connectivity Map (Cmap) was used to screen the interaction drugs with atorvastatin.

Results Compared with the control cells, 649 genes were detected to have marked changes with 295 up-regulated and 354 down-regulated expression in atorvastatin treated EA. hy 926 cells. 13 pathways including coagulation in upregulated gene expression and 113 pathways mainly enrichment in cell cycle in downregulated gene expression using GSEA showed statistical significance. In Cmap analysis the profile of the HDAC inhibitor MS-275, trichostatin A, Vorinostat and resveratrol, Phenothiazines derivatives were found to be highly similar to that of atorvastatin $(p<0.001)$.

Conclusions The study of drug interactions based on the bioinformatics is feasible, atorvastatin have a synergistic effect with histone deacetylase inhibitors, resveratrol and phenothiazine derivatives. 